\title{
Relação entre capacidade funcional e capacidade de tomada de decisão em longevos
}

\author{
Relationship between physical function and decision-making capacity \\ in oldest-old
}

\author{
Andrea Ribeiro Mirandola ${ }^{a}$, Angelo José Gonçalves Bós ${ }^{b}$ \\ - Instituto de Geriatria e Gerontologia da PUCRS. <armirandola@gmail.com> \\ bInstituto de Geriatria e Gerontologia da PUCRS. < angelo.bos@pucrs.br>
}

\section{ARTICLE INFO}

\section{Article history}

Received: 25/11/2015

Accepted: 22/01/2016

\section{Correspondent Author}

Angelo José Gonçalves Bós

Instituto de Geriatria e Gerontologia da PUCRS

Av. Ipiranga 6681, prédio 81 , sala 703

91530-001 Porto Alegre, RS, Brasil

<angelo.bos@pucrs.br>

\section{(C) 2015 All rights reserved}

Editors

Alfredo Cataldo Neto

Irenio Gomes

\begin{abstract}
RESUMO
Introdução: $\bigcirc$ envelhecimento populacional é um desafio deste século, impactando a sociedade e provocando necessidades de ajustes estruturais. A longevidade envolve inúmeras alterações na vida do idoso e quando associada à incapacidade funcional pode comprometer a funcionalidade, saúde, privando-o de uma vida autônoma e saudável. Objetivo: Observar a relação entre capacidade funcional e capacidade de tomar decisão em longevos. Métodos: Trata-se de uma pesquisa quantitativa descritiva analítica transversal, em longevos (80 anos ou mais) do Rio Grande do Sul. Os instrumentos utilizados foram questionário geral, questionário de interação entre o longevo e sua vida, medidas de independência funcional e capacidade de tomar decisão. Resultados: A amostra foi composta por 47 longevos, divididos em octogenários (19) e nonagenários (28). A fase psicológico-moral dos octogenários foi classificada em média como conscienciosa e dos nonagenários como conformista. Os grupos apresentaram maior perda nas decisões relacionadas a atividades no lar. Os nonagenários apresentaram pior capacidade funcional quando comparados aos octogenários, essa observação foi menos acentuada em longevos que se encontravam em fase conscienciosa do desenvolvimento psicológico moral, ou seja, com maior capacidade de tomar decisões. Conclusões: Observamos uma relação positiva entre capacidade funcional e capacidade de tomada de decisão nos longevos estudados. Investigar os fatores que possam contribuir para que o longevo tenha maior independência e autonomia é fundamental para a reestruturação dos serviços, planejar ações de saúde pública e prestar atendimento com dignidade, buscando por maior tempo possível à manutenção da qualidade de vida em longevos. PALAVRAS-CHAVE: Idosos de 80 anos ou mais; Autonomia pessoal; Classificação internacional da funcionalidade; Incapacidade e saúde.
\end{abstract}

\begin{abstract}
Introduction: The aging population is a challenge of this century, affecting society and imposing needs of structural adjustments. The longevity involves numerous changes in the lives of the elderly and when associated with functional disability may impair functionality, health, precluding an autonomous and healthy life. Objective: To observe the relationship between functional capacity and ability to make decisions of the oldest old. Methods: This study was descriptive analytical cross-sectional quantitative research in the oldest-old ( 80 years or more) of RS. The instruments used were a general questionnaire, a survey of interaction between the oldest-old and his/her life, assessment of functional independence and ability to make decisions. Results: The sample consisted of 47 oldest-old, divided in octogenarians (19) and nonagenarians (28). The moral psychological phase of octogenarians was classified on average as conscientious and nonagenarians as conformist. Both groups showed greater loss in decisions related to activities at home. Nonagenarians had worse functional capacity when compared to octogenarians. However, nonagenarians with higher moral psychological phase were much closer to the functional capacity of the octogenarians. Conclusions: We observed a positive relationship between physical function and decision-making capacity in oldest-old. Investigating factors that may contribute to the oldest-old maintain higher quality of life is essential for the restructuring of services, public health action plan and providing care with dignity. This could be achieved by maintaining functional independence as long as possible, preserving or recovering autonomy and always give priority to a better quality of life of seniors in the final stage of their lives.

Keywords: Aged, 80 years and overs, personal autonomy, International classification of functioning, disability, and health.
\end{abstract}




\section{INTRODUÇÃO}

O envelhecimento populacional é um dos desafios mundiais do século XXI assim como no Brasil. Nos últimos anos o país vem apresentando um novo padrão demográfico que se caracteriza pela redução da taxa de crescimento populacional e por transformações profundas na composição de sua estrutura etária. Observa-se também um significativo aumento do contingente de idosos, impactando a sociedade e consequentemente provocando necessidades de ajustes nas estruturas físicas e sociais de acordo com as demandas da população. ${ }^{1}$

O Instituto Brasileiro de Geografia e Estatística (IBGE) ${ }^{2}$ estima que em 2014 existam 23 milhões de idosos no País. Já no ano de 2060 é esperado um número aproximado de 73,6 milhões de idosos, representando um aumento de aproximadamente $220 \%$ de idosos. Em relação aos longevos (80 anos ou mais) o número também vem aumentando de forma muito acelerada, constituindo o segmento populacional que mais cresce nos últimos tempos, estima-se chegar a 3,2 milhões de longevos no ano de 2014 e aproximar-se a 19 milhões no ano 2060, apontando um crescimento de 500\% deste segmento populacional.

A longevidade está associada ao processo do envelhecimento e sua consequência, a velhice, e continua sendo umas das preocupações da humanidade, principalmente quando associada à incapacidade funcional. ${ }^{3}$ As inúmeras alterações na vida do idoso, podem comprometer a funcionalidade, mobilidade, saúde, privando-o de uma vida autônoma e saudável. ${ }^{4}$

Desta forma, o maior desafio na atenção à pessoa idosa é conseguir contribuir para que, mesmo com o aparecimento de progressivas limitações, elas possam redescobrir possibilidades de viver sua própria vida com autonomia e qualidade. Essa possibilidade aumenta na medida em que a sociedade considera o contexto familiar e social e consegue reconhecer as potencialidades e o valor das pessoas idosas. ${ }^{3}$

Embora o Brasil seja um dos países com maior crescimento da faixa etária muito idosa é escasso o número de pesquisas no país voltadas para esse segmento populacional. ${ }^{5}$ Portanto, diante da relevância do tema, do aumento rápido e progressivo das pessoas longevas e a escassez de artigos nessa área, faz-se necessário conhecer as características desses longevos para planejar ações de saúde pública voltadas a este segmento populacional. O objetivo do trabalho foi observar a relação entre capacidade funcional e capacidade de tomada de decisão em longevos.

\section{METODOLOGIA}

Trata-se de uma pesquisa quantitativa descritiva analítica e transversal que envolveu longevos (80 anos de idade ou mais) assistidos pelo Ambulatório Multiprofissional de Atenção aos Longevos (AMPAL) do Serviço de Geriatria do Hospital São Lucas da Pontifícia Universidade Católica do Rio Grande do Sul (PUCRS) e da Associação dos Ferroviários Sul Riograndenses (AFSR).

A amostra foi composta de 47 longevos divididos, por faixa etária, em dois grupos: grupo de nonagenários e grupo de octogenários. O grupo dos nonagenários foi composto por 28 longevos e grupo dos octogenários foi composto por 19 longevos.

Os longevos foram convidados a participar do estudo e receberam, individualmente, as informações referentes aos procedimentos aos quais seriam submetidos. A coleta de dados foi realizada por meio de entrevista e aplicação dos seguintes instrumentos: Questionário geral, Questionário de interação entre o longevo e sua vida, Medida de Independência Funcional (MIF) e Questionário sobre a capacidade de tomada de decisão (IDPM). O longevo que não teve condições cognitivas de responder sozinho, fato esse observado pelo entrevistador pela falta de coerência nas respostas iniciais do questionário, o IDPM foi excluído na análise deste parâmetro.

Os autores perguntaram aos longevos se, comparado há 10 anos pregressos, houve mudanças na frequência com que decide sobre o seu autocuidado, saúde, atividades sociais e lazer. $O$ instrumento MIF foi traduzido e adaptado ${ }^{6}$ no Brasil e avalia a independência funcional para a realização de tarefas motoras e cognitivas. No presente trabalho foram avaliadas as 13 tarefas motoras divididas em quatro domínios: autocuidado, controle esfincteriano, transferência e locomoção. Cada tarefa é pontuada de 1 (dependência total) a 7 (independência), o somatório das tarefas em cada domínio foi padronizado para $100 \%$ de independência. O questionário IDPM foi validado por Raymundo \& Goldim ${ }^{7}$ e está relacionado com capacidade de tomar decisões ao classificar os participantes em uma das sete fases do desenvolvimento psicológico-moral: pré-social, impulsiva, oportunista, conformista, conscienciosa, autônoma e integrada.

A MIF e o IDPM foram considerados variáveis dependentes e tratadas tanto quantitativamente quanto qualitativamente. A MIF foi analisada pelos 4 domínios e total. O IDPM foi classificado conforme a fase de desenvolvimento psicológico moral. As variáveis independentes potenciais fatores de confusão 
do estudo foram: idade, sexo, raça, escolaridade, estado civil, renda do idoso, presença de cuidador, número de filhos e multimorbidades.

No tratamento dos dados socioeconômicos e demográficos, para as variáveis quantitativas (idade, renda e tempo de estudo) foram realizadas análises descritivas com média e desvio-padrão inicialmente para cada nível da faixa etária e também para as médias do IDPM e MIF. Para as variáveis qualitativas (classificação do IDPM, estado conjugal) foram analisadas por frequências e percentuais.

As médias das variáveis dependentes foram comparadas entre os níveis de raça e o estado conjugal pela análise de variância (ANOVA). A correlação das variáveis dependentes e idade, anos de estudo, renda familiar, número de filhos vivo e multimorbidades foram testadas pela regressão linear. A correlação entre idade e capacidade funcional para cada fase do desenvolvimento psicológico moral foram observadas por meio da construção de gráficos de dispersão.

Níveis de significância menores do que 5\% $(p<0,05)$ foram considerados significativos. Níveis de significância entre 5 e 10\% foram considerados indicativos de significância. ${ }^{8}$

O presente estudo foi aprovado pelo Comitê de Ética em Pesquisa da PUCRS, parecer no 107.901 em 14/09/2012. Todos os participantes assinaram o Termo de Consentimento Livre e Esclarecido.

\section{RESULTADOS}

A amostra foi composta de 47 longevos divididos, por faixa etária, em dois grupos: grupo de nonagenários e grupo de octogenários. A Tabela 1 apresenta a distribuição percentual do sexo, estado conjugal e raça, segundo faixa etária. $\mathrm{O}$ grupo dos nonagenários foi composto por 28 longevos sendo 20 mulheres $(71,4 \%)$ e oito homens $(28,6 \%)$, enquanto o grupo dos octogenários apresentou 19 longevos, 13 mulheres $(68,4 \%)$ e seis homens $(31,6 \%)$. Em relação ao estado conjugal 24 eram viúvos $(85,7 \%)$, três eram casados $(10,7 \%)$ e um solteiro $(3,6 \%)$; em relação a raça 24 longevos são brancos $(85,7 \%)$, dois pardos $(7,1 \%)$ e dois negros $(7,1 \%)$.

O grupo dos octogenários foi composto por 19 longevos sendo que 13 eram mulheres $(68,4 \%)$ e seis homens $(31,6 \%)$, destes 13 eram viúvos $(68,4 \%)$ e seis casados $(31,6 \%)$; em relação à raça 11 longevos referiram ser da cor branca $(57,9 \%)$, seis pardos $(31,6 \%)$ e dois negros $(10,5 \%)$. Em relação ao sexo, todas as mulheres eram viúvas e a maioria dos homens $(64,3 \%)$ era casado.

O grupo de nonagenários apresentou uma idade média de 93,6 anos $( \pm 2,8)$, média da renda familiar de $R \$ 2.375,92( \pm 1.754,99)$ e tempo de estudo de $3,4$ anos $( \pm 1,9)$ (Tabela 1$)$.

A Tabela 2 apresenta as médias do IDPM e Capacidade Funcional, medidos por meio do MIF segundo faixa etária.

O grupo de nonagenários apresentou uma média de 3,9 $\pm 1,7$ no IDPM, que classifica a média dos longevos deste grupo como Conformista, ou seja, as crenças do indivíduo se sobrepõem aos seus próprios desejos e justificam as contingências impostas pelo meio. Deste grupo quatro longevos não conseguiram responder.

Tabela 1. Distribuição sociodemográfica dos participantes segundo a faixa etária. Porto Alegre, RS. 2014

\begin{tabular}{|c|c|c|c|c|}
\hline \multirow{2}{*}{ Variáveis } & \multicolumn{2}{|c|}{ Grupos } & \multirow{2}{*}{ Total } & \multirow{2}{*}{$\mathbf{p}$} \\
\hline & Nonagenários & Octogenários & & \\
\hline $\begin{array}{l}\text { Sexo }(\mathrm{n}) \\
\text { Feminino } \\
\text { Masculino }\end{array}$ & $\begin{array}{l}71,4 \%(20) \\
28,6 \%(08)\end{array}$ & $\begin{array}{l}68,4 \%(13) \\
31,6 \%(06)\end{array}$ & $\begin{array}{l}70,2 \%(33) \\
29,8 \%(14)\end{array}$ & 0,8249 \\
\hline $\begin{array}{l}\text { Estado conjugal (n) } \\
\text { Casado } \\
\text { Viúvo } \\
\text { Solteiro }\end{array}$ & $\begin{array}{r}10,7 \%(03) \\
85,7 \%(24) \\
3,6 \%(01)\end{array}$ & $\begin{array}{c}31,6 \%(06) \\
68,4 \%(13) \\
0,0 \%(00)\end{array}$ & $\begin{array}{r}19,1 \%(09) \\
78,7 \%(37) \\
2,2 \%(01)\end{array}$ & 0,1551 \\
\hline $\begin{array}{l}\text { Raça (n) } \\
\text { Branca } \\
\text { Parda } \\
\text { Negra }\end{array}$ & $\begin{array}{r}85,7 \%(24) \\
7,1 \%(02) \\
7,1 \%(02)\end{array}$ & $\begin{array}{l}57,9 \%(11) \\
31,6 \%(06) \\
10,5 \%(02)\end{array}$ & $\begin{array}{r}74,4 \%(35) \\
17,0 \%(08) \\
8,6 \%(04)\end{array}$ & 0,0318 \\
\hline Idade (anos)* & $93,6 \pm 2,8$ & $85,3 \pm 2,6$ & $90,3 \pm 4,9$ & $<0,0001$ \\
\hline Renda familiar (mil reais)* & $2,3 \pm 1,75$ & $2,1 \pm 1,44$ & $2,3 \pm 1,6$ & 0,6172 \\
\hline Tempo de estudo (anos) & $3,4 \pm 1,9$ & $3,7 \pm 2,2$ & $3,5 \pm 2,1$ & 0,5804 \\
\hline
\end{tabular}

* Média \pm desvio-padrão. 
Tabela 2. Médias do Instrumento de Desenvolvimento Psicológico-Moral (IDPM) e capacidade funcional (MIF) segundo faixa etária. Porto Alegre, RS. 2014.

\begin{tabular}{l|cc|c}
\hline \multicolumn{1}{|c|}{ Variáveis } & \multicolumn{2}{c}{ Grupos } & \\
& $\begin{array}{c}\text { Nonagenários } \\
\text { (média } \pm \text { dp) }\end{array}$ & $\begin{array}{c}\text { Octogenários } \\
\text { (média } \pm \text { dp) }\end{array}$ & p \\
\hline IDPM (42)* & $3,9 \pm 1,7$ & $4,3 \pm 1,2$ & 0,4139 \\
MIF (47) & $38,5 \pm 5,54$ & $41,8 \pm 0,6$ & 0,0125 \\
Autocuidado & $13,2 \pm 1,15$ & $13,3 \pm 0,7$ & 0,9206 \\
Controle esfíncteres & $17,8 \pm 4,4$ & $20,8 \pm 0,6$ & 0,0061 \\
Mobilidade & $11 \pm 3,5$ & $13,4 \pm 1,2$ & 0,0065 \\
Locomoção & $80,6 \pm 13,1$ & $89,4 \pm 2,7$ & 0,0066 \\
\hline Total motor & & & \\
\hline
\end{tabular}

* Cinco participantes não responderam o IDPM.

O grupo de octogenários apresentou uma média ficou em 4,3 $\pm 1,2$, mostrando que a média os classifica na fase Conscienciosa, demonstrando que o indivíduo é capaz de tomar decisões de forma mais autônoma, cotejando os desejos e as crenças, mas, ainda é "passível de constrangimento" conforme Raymundo \& Goldim? Deste grupo um longevo não conseguiu responder o instrumento.

A MIF motora é mensurada por meio de quatro domínios. $\mathrm{O}$ grupo de nonagenários teve uma média de 38,5 $\pm 5,54$ no domínio autocuidado, que considera as atividades diárias básicas como alimentação, higiene pessoal, banho, vestir a roupa, no domínio controle esfíncteres, ou seja, manter controle sobre urina e evacuação a média ficou em 13,2 $\pm 1,15$, no domínio mobilidade que contempla transferências a média foi de $17,8 \pm 4,4$, e no domínio locomoção relacionada com caminhar e subir escadas a média foi de $11,0 \pm 3,5$, apresentando um MIF motora total de 80,6 $\pm 13,1$. O grupo de octogenários apresentou uma média de $41,8 \pm 0,6$ no autocuidado, $13,3 \pm 0,7$ no controle de esfíncteres, 20,8 $\pm 0,6$ na mobilidade e $13,4 \pm 1,2$ na locomoção, apresentando um valor total na escala motora MIF de 89,4 42,7 , conforme Tabela 2.

Ambos os grupos foram considerados independentes (sem ajuda), na média geral do MIF. Os nonagenários foram classificados na Independência Modificada, o que significa que a atividade requer uma ajuda técnica, adaptação, prótese ou órtese, um tempo de realização demasiado elevado, ou não pode ser realizado em condições de segurança suficientes. Já os octogenários se aproximaram da Independência Completa, onde todas as tarefas descritas que constituem a atividade em questão são realizadas em segurança, sem modificação, sem ajuda técnica e em tempo razoável.

O grupo de octogenários apresentou maior independência funcional, com diferença significativa nos domínios autocuidado, mobilidade, locomoção e na soma total dos domínios motores do MIF em relação ao grupo de nonagenários, conforme Tabela 2.

A Tabela 3 apresenta a distribuição percentual da diminuição da frequência que o longevo decide sobre suas atividades comparado há 10 anos pregressos, segundo faixa etária.

Tabela 3. Distribuição percentual da diminuição da frequência que o longevo decide sobre suas atividades comparadas há 10 anos pregressos, segundo faixa etária. Porto Alegre, RS. 2014.

\begin{tabular}{|l|c|c|c}
\hline \multicolumn{1}{|c}{ Variáveis } & \multicolumn{2}{c}{ Grupos } & Total \\
\cline { 2 - 4 } & $\begin{array}{c}\text { Nonagenários } \\
(\mathbf{n = 2 8 )}\end{array}$ & $\begin{array}{c}\text { Octogenários } \\
(\mathbf{n = 1 9 )}\end{array}$ & \\
\hline Decisão autocuidado & $32,1 \%(09)$ & $10,5 \%(02)$ & $23,4 \%(11)$ \\
Decisão no lar & $60,7 \%(17)$ & $31,6 \%(06)$ & $48,9 \%(23)$ \\
Decisão saúde & $39,3 \%(11)$ & $10,5 \%(02)$ & $27,7 \%(13)$ \\
Decisão social & $64,3 \%(18)$ & $15,8 \%(03)$ & $44,7 \%(21)$ \\
\hline
\end{tabular}

No que diz respeito à frequência de tomada de decisão sobre suas atividades, comparando hoje a 10 anos pregressos o grupo de nonagenários apresentaram os seguintes resultados: nove longevos $(32,1 \%)$ diminuíram a frequência de decisão em relação a atividades de autocuidado, 17 longevos (60,7\%) diminuíram a frequência de decisão em relação a atividades desenvolvidas no lar, 11 longevos (39,3\%) diminuíram a frequência de decisão em relação a questões sobre sua saúde e 18 longevos (64,3\%) diminuíram a frequência de decisão em relação a atividades sociais. No grupo de octogenários dois longevos $(10,5 \%)$ diminuíram a frequência de decisão em relação ao autocuidado, seis longevos $(31,6 \%)$ diminuíram a frequência de decisão em relação a atividades desenvolvidas no lar, dois longevos $(10,5 \%)$ diminuíram a frequência de decisão em relação a questões sobre sua saúde e três longevos $(15,8 \%)$ diminuíram a frequência de decisão em relação atividades sociais. Os dois grupos apresentaram maior perda nas frequências de decisão relacionadas a atividades desenvolvidas no lar, seguida das decisões sociais. Os resultados estão apresentados na Tabela 3.

A Figura 1 relaciona capacidade funcional, fase psicológica moral e idade e mostra que o grupo que não soube responder o IDPM apresentou uma linha acentuadamente descendente que as demais fases, demonstrando que a idade exerce uma influência negativa sobre a capacidade funcional de uma forma mais importante neste grupo. O grupo da fase autônoma apresentou uma linha ascendente. Os grupos das fases 


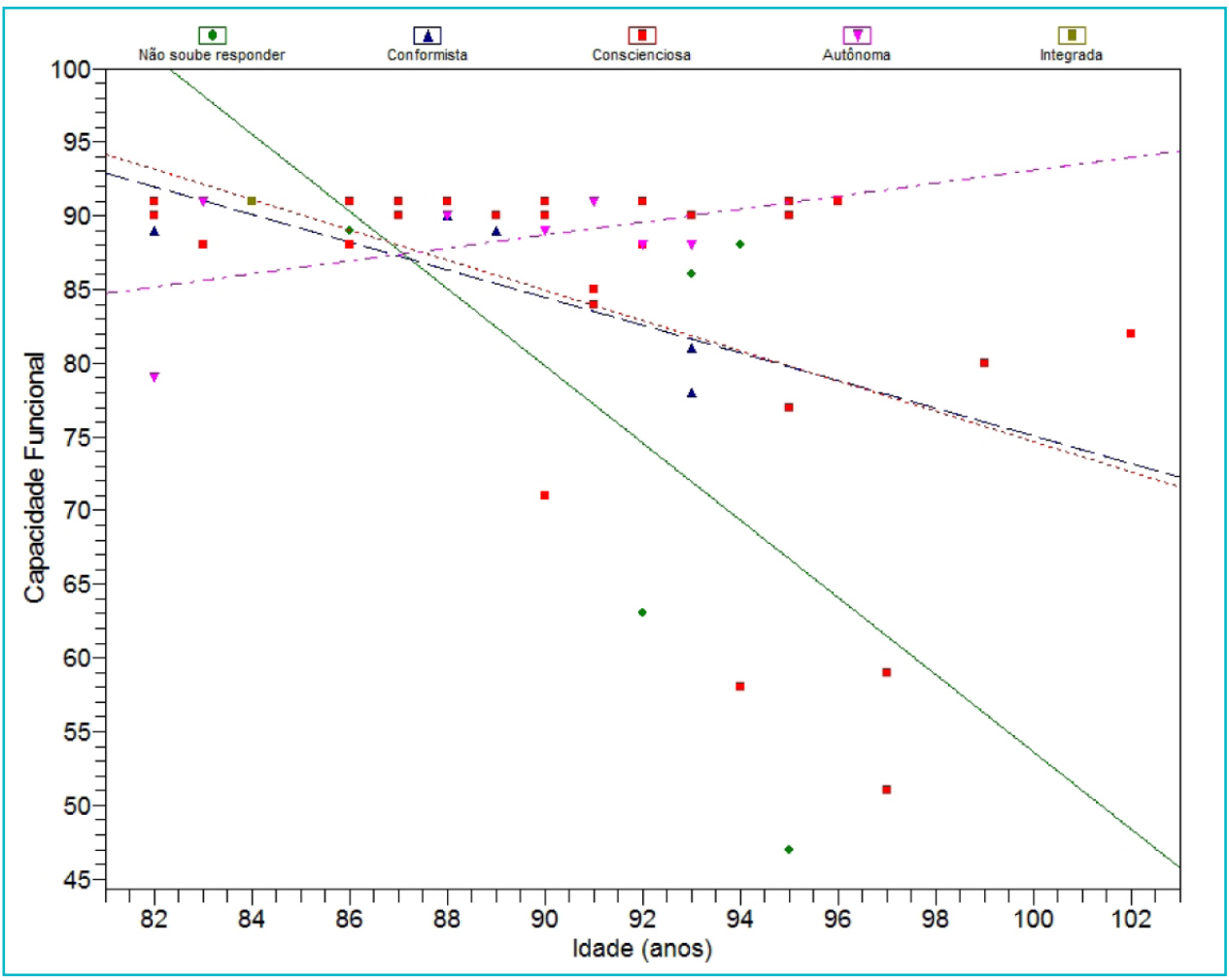

Figura 1. Relação da capacidade funcional, fase psicológica-moral e idade. Porto Alegre, RS. 2014.

conscienciosa e conformista apresentaram linhas muito semelhantes. A interpretação do gráfico sugere que quanto maior a fase do desenvolvimento psicológico moral melhor é a relação da idade com a Capacidade Funcional, avaliado por meio da MIF.

\section{DISCUSSÃO}

O presente estudo teve o objetivo de estudar a relação entre capacidade funcional e capacidade de tomada de decisão em longevos. A amostra foi composta por longevos de 80 anos ou mais de idade.

A capacidade funcional surge como um novo paradigma na atenção à saúde da pessoa idosa, assim como a autonomia que também é meta a ser alcançada quando se fala em saúde de idosos e longevos, na medida em que esses domínios são os melhores indicadores de saúde na velhice.

A amostra estudada constitui-se na maior parte por longevos do sexo feminino (70\%). Isto reflete a realidade brasileira e está de acordo com o descrito por Bird et al., ${ }^{9}$ por ser uma das características do processo de envelhecimento populacional.

Um estudo que corrobora esse achado analisou as características de saúde e socioeconômicas de idosos longevos em duas cidades distintas, em Ribeirão
Preto-SP e Caxias do Sul-RS, demonstrando que em ambas as cidades, o sexo feminino predomina entre os idosos longevos. ${ }^{10}$ Em outros estudos ${ }^{11,12}$ evidenciou-se o maior número de mulheres idosas em pesquisas confirmando a feminilização do envelhecimento. A feminilização do envelhecimento tem sido relacionada à maior longevidade observada nas mulheres, ${ }^{13}$ mas também está relacionada à maior participação das mulheres em atividades sociais ${ }^{11} \mathrm{e}$ busca pelos serviços de saúde.

No que diz respeito ao estado conjugal, todas as mulheres desse estudo eram viúvas, podendo-se inferir que o aumento do número de idosas viúvas dentro desta faixa etária está ligado à maior longevidade das mulheres. Estas tendem a permanecer sozinhas, enquanto, os homens procuram por um novo casamento. ${ }^{14}$

Os anos de estudo dos longevos participantes desta pesquisa ficaram entre três e quatro anos tanto em octogenários quanto em nonagenários, demonstrando uma baixa escolaridade, dados semelhantes foram encontrados nos estudos de Feliciano et al. ${ }^{15} \mathrm{e}$ Lourenço, ${ }^{16}$ indicando que os idosos, principalmente os longevos, apresentam poucos anos de estudo. Desta forma, os longevos pesquisados, representando pacientes do AMPAL e da AFSR, apresentavam nível 
de escolaridade semelhante ao da população longeva brasileira. Pode-se dizer que a baixa escolaridade é um reflexo da organização social do início do século passado, que dificultava o acesso ao ensino formal. ${ }^{16}$

No presente estudo observamos que a idade é fator importante na capacidade funcional, já que os longevos dos grupos de octogenários apresentaram maior independência funcional, com diferença significativa nos domínios autocuidado, mobilidade, locomoção e na soma total motora dos domínios da MIF quando comparado aos integrantes do grupo dos nonagenários. Estudos ${ }^{17-18} \mathrm{com}$ idosos e longevos mostraram que idosos mais velhos apresentavam pior capacidade funcional, assim também entre os próprios longevos, na medida em que a idade aumenta ocorre um declínio da capacidade funcional, resultados estes que corroboram os achados do presente estudo.

O questionário de interação entre o longevo e sua vida, foi desenvolvido pelos autores do presente estudo com objetivo de captar a frequência com que o longevo decide sobre suas atividades, comparando hoje a 10 anos pregressos. Observou-se que os integrantes dos dois grupos apresentaram maior perda nas frequências de tomadas de decisão relacionadas a atividades desenvolvidas no lar, como preparo e escolha da comida, rotinas em relação a ouvir rádio, assistir TV, fazer leitura, atender ou fazer ligação telefônica. De acordo com Baltes et $\mathrm{al}^{19}$ a perda de domínio sobre o próprio ambiente pode ter consequências negativas sobre a saúde física e psicológica do indivíduo, deste modo, os ambientes devem ser estruturados para serem estimuladores e exigentes quanto às forças $e$ competências do idoso, no caso o longevo, para garantir as necessidades básicas e universais como segurança e autonomia.

O segundo domínio com maior perda de tomada de decisão foi o social que se refere às atividades relacionadas à vida social do longevo, como visita a amigos e familiares, passeios, compras de alimentos e produtos pessoais, participação em grupos. Estudo de Nogueira et al. ${ }^{20}$ aponta que o isolamento social pode comprometer a capacidade funcional $\mathrm{e}$ consequentemente interferir na qualidade de vida e capacidade de decisão. Portanto é importante estimular o longevo para decidir sobre as questões que se referem a sua vida.

Acredita-se ser importante pesquisar sobre a tomada de decisão dos longevos, pois este é um atributo que pode contribuir para que o mesmo tenha maior qualidade de vida, destacando que com muita frequência a limitação física é muitas vezes confundida com a impossibilidade para tomada de decisão. Goldim ${ }^{21}$ refere ser fundamental desvincular as perdas físicas das cognitivas e ser necessário valorizar os ganhos positivos da idade, como a sabedoria e experiência.

No presente estudo foi aplicado o Instrumento de Desenvolvimento Psicológico Moral (IDPM) que tem sido utilizado para avaliar a capacidade de tomada de decisão ao observar em que fase do desenvolvimento psicológico moral o indivíduo se encontra, neste caso, os longevos. $\mathrm{O}$ grupo de nonagenários foi classificado como conformista, o grupo dos octogenários apresentaram uma média melhor sendo classificado na fase conscienciosa. Da amostra total de longevos, cinco não conseguiram responder, o que se entendeu como total falta de capacidade de tomar decisão.

Pesquisas realizadas com idosos que utilizaram o IDPM $^{22,23}$ apresentaram resultados semelhantes, nas quais a maioria da amostra também se concentrou nas fases conscienciosa e conformista, corroborando os resultados da presente pesquisa.

A importância de pesquisar sobre a capacidade de tomada de decisão foi observada também no estudo de Celich. ${ }^{24}$ Esse autor concluiu que a autonomia é um dos determinantes para uma boa qualidade de vida e um dos pressupostos básicos para a tomada de decisão. Demonstrando o valor deste atributo na busca dos fatores que contribuem para uma vida com qualidade.

No presente estudo observou-se que quanto maior a fase do desenvolvimento psicológico moral do longevo, melhor é a relação da idade com a capacidade funcional. Aparentemente os longevos com maior fase do desenvolvimento psicológico moral compensam a condição física imposta pela idade de uma forma mais eficiente.

Constatou-se durante a revisão de literatura um acentuado déficit de estudos que abordam o tema em questão, principalmente em idosos de 80 anos ou mais, ou seja, os longevos. Esta constatação dificultou a discussão dos resultados em alguns aspectos levantados no estudo, portanto, deve-se considerar uma limitação na comparação de estudos com resultados semelhantes, tendo em vista os diferentes protocolos, além de diferenças socioculturais entre os grupos pesquisados. Deste modo, há um vasto campo de pesquisa para ser explorado pelos profissionais de saúde, que é o estudo das questões que envolvem longevos.

\section{CONSIDERAÇÕES FINAIS}

$\mathrm{Na}$ comparação que pode ser realizada entre octogenários e nonagenários observa-se diferenças significativas na capacidade funcional, entretanto, a capacidade de tomada de decisão não apresentou diferença significativa. $O$ estudo aponta que a diminuição da capacidade funcional está relacionada 
à idade. Com isso, o foco de atenção ao longevo deve ser integral e as ações a saúde precisam ser efetivadas, aliando o incentivo à pesquisa com ações planejadas e direcionadas para com vistas a melhoria das condições de saúde e qualidade de vida deste segmento populacional.

Os resultados deste estudo mostraram que a capacidade de decisão do longevo diminuiu com avançar da idade, assim como a frequência de decisão de atividades desenvolvidas no lar e decisões relacionadas às atividades sociais. A capacidade de tomar decisão é um atributo importante na vida do longevo, assim sendo é imprescindível estudar acerca dos atributos que contribuem para que o longevo tenha maior qualidade de vida. Observou-se ser importante a criação e aplicação do questionário de interação entre o longevo e sua vida, pois se acredita ter sido eficiente ao captar o quanto o longevo pode perder em relação à capacidade de decisão nas atividades de sua vida.

\section{REFERÊNCIAS}

1. Instituto Brasileiro de Geografia e Estatística (IBGE). Disponível em: < http://www.sidra.ibge.gov.br/bda/tabela/ listabl.asp $? z=c d \& o=3 \& i=P \& c=3107>$. Acesso em: 05 jun. 2015.

2. Instituto Brasileiro de Geografia e Estatística (IBGE). Projeção da população do Brasil por sexo e idade para o período 2000-2060 Disponível em: < http://www.ibge.gov.br/ home/estatistica/populacao/projecao da populacao/2013/ default tab.shtm >. Acesso em: 14 jun. 2015.

3. BRASIL. Ministério da Saúde. Envelhecimento e saúde da pessoa idosa. Caderno de Atenção Básica, n. 19. Brasília. 2006.

4. Karinkanta S, Heinonen A, Sievänen H, Uusi-Rasi K, Kannus P. Factors predicting dynamic balance and quality of life in home-dwelling elderly women. Gerontology. 2005; 51(2):116-21.

5. Rosset I, Pedrazzi EC, Roriz-Cruz M, Morais EP, Rodrigues RAP. Tendências dos estudos com idosos mais velhos na comunidade: uma revisão sistemática (inter)nacional. Rev Esc Enferm USP. 2011;45(1):264-71.

6. Riberto M, Miyasaki H M, Jucá S S H, Sakamoto H, Pinto P P N, Battistella. Validação da versão brasileira da Medida de Independência Funcional. Acta Fisiátrica. São Paulo. 2004;11(2)72-6.

7. Raymundo MM, Goldim JR. Moral-psychological development related to the capacity of adolescents and elderly patients to consent. Journal of Medical Ethics. 2008;34:602-5.

8. Bós AJG. Epi Info ${ }^{\circledR}$ sem mistérios: um manual prático [recurso eletrônico] / Dados eletrônicos - Porto Alegre: EDIPUCRS, 2012. 211 p. Sistema requerido: Adobe Acrobat Reader. Disponível em: < http://ebooks.pucrs.br/edipucrs/ epiinfo.pdf> Acesso: 14 jun. 2015.

9. Bird C, Shugarman L, Lynn J. Age and gender differences in health care utilization and spending for medcare beneficiaries in their last years of life. J Palliat Med. 2002 oct.;5(5):705-12.
10. Rodrigues RAP, Rosset I, Roriz-Cruz M, Santos JLF, Haas VJ, Wehbe SCCF. Diferenciais Sócio-Econômicos e de Saúde Entre duas Comunidades de Idosos Longevos. Rev Saúde Pública. 2011;45(2):391-400.

11. Silva LAA, Leite MT, Winck MT, Hildebrandt LM, Kirchner RM. Qualidade de vida e nível cognitivo de pessoas idosas participantes de grupos de convivência. Rev. Bras. Geriatr. Gerontol. 2012;15(3):481-92.

12. Victor JF, Ximenes LB, Almeida PC, Vasconcelos FF. Perfil sociodemográfico e clínico de idosos atendidos em Unidade Básica de Saúde da Família. Acta Paul Enferm. 2009 ago.; 22(1):49-54.

13. Fernandes MGM, Nascimento NFS, Costa KNFM. Prevalência e determinantes de sintomas depressivos em idosos atendidos na atenção primária de saúde. Rev Rene. $2010 \mathrm{jan} / \mathrm{mar} ; 11(1): 19-27$.

14. Lima-Costa M F; Camarano AA. Demografia e epidemiologia do envelhecimento no Brasil. In: Moraes EN. Princípios básicos de geriatria e gerontologia. Belo Horizonte: Coopmed, 2008.

15. Feliciano AB, Moraes AS, Freitas ICM. O perfil do idoso de baixa renda no município de São Carlos, São Paulo, Brasil: um estudo epidemiológico. Cad Saude Publica. 2004 nov./ dez.;20(6):1575-85.

16. Lourenço, TM. Capacidade funcional do idoso longevo em unidades de internação hospitalar na cidade de Curitiba-PR. [Dissertação]. Curitiba: Universidade Federal do Paraná; 2011.

17. Scattolin FAA, Colombo RCR, Diogo MJD. Preditores de independência funcional em idosos portadores de insuficiência cardíaca. Acta Fisiatrica. 2007 dez.;14(4): 219-25.

18. Maria CR, Nunes RCL. Ribeiro LEF, Rosado PL, Franceschini SC. Influência das características sociodemográficas e epidemiológicas na capacidade funcional de idosos residentes em Ubá, Minas Gerais. Rev Bras Fisioter. 2009 set./out.;13(5):376-82.

19. Baltes M, Silverberg S. A dinâmica dependência-autonomia no curso de vida. In: Neri A, org. Psicologia do Envelhecimento. São Paulo: Papirus; 1995.

20. Nogueira S, Ribeiro RCL, Rosado LPEL, Franceschini SCC, Ribeiro AQ, Pereira AQ. Fatores determinantes da capacidade funcional em idosos longevos. Rev. Bras Fisioter. 2010;14(4):322-9.

21. Goldim JR. Bioética e envelhecimento. In: Freitas EV, Py L, Néri AL, Cançado FAX, Gorzoni ML, Rocha SM. Tratado de geriatria e gerontologia. Rio de Janeiro: Guanabara Koogan; 2002. p. 85-99.

22. Raymundo, MM. Avaliação da diversidade no processo de obtenção da autorização por representação em situações assistenciais e de pesquisa em crianças e idosos. [Tese]. Porto Alegre: Universidade Federal do Rio Grande do Sul; 2007.

23. Pavan, AP. Avaliação da qualidade de vida e tomada de decisão em idosos participantes de grupos socioterápicos da cidade de Arroio do Meio, RS, Brasil. [Dissertação]. Porto Alegre: Pontifícia Universidade Católica do Rio Grande do Sul; 2008.

24. Celich, KLS. Domínios de qualidade de vida e capacidade para a tomada de decisão em idosos participantes de grupos da terceira idade. [Dissertação] Porto Alegre: Pontifícia Universidade Católica do Rio Grande do Sul; 2008. 